\title{
INCREASE OF INTRACELLULAR BAFF IN B CELLS OF SJÖGREN'S PATIENTS IS NOT AFFECTED BY DECREASE OF BAFFR
}

\author{
Jan Krejsek ${ }^{1}$, Martina Koláčková ${ }^{1}$, Irena Lindrováá, Radovan Slezák ${ }^{2}$, Ctirad Andrýs ${ }^{1}$ \\ Charles University in Prague, Faculty of Medicine and University Hospital in Hradec Králové, Czech Republic: Department \\ of Clinical Immunology and Allergology ${ }^{1}$; Charles University in Prague, Faculty of Medicine and University Hospital in \\ Hradec Králové, Czech Republic: Department of Stomatology²
}

\begin{abstract}
Summary: The presence of a broad spectrum of autoantibodies in Sjögren's syndrome ( $\mathrm{SjS}$ ) patients is the result of abnormal B-cell regulation that can be at least partially explained by abnormal BAFF/BAFFR regulation. The objective of this study was to determine both membrane and intracellular expression of BAFF/BAFFR in monocytes and B-cells in peripheral blood of 19 primary Sjögren's syndrome patients and 20 healthy controls using flow cytometry. We also measured sBAFF in serum. Compared to healthy controls, both surface and intracellular expression of BAFF was significantly increased in monocytes and B-cells of SjS patients. Also serum sBAFF level was elevated. Expression of BAFFR on B-cells of SjS patients was surprisingly decreased, but there was no clear increase or decrease within monocytes. Our results indicate that activated monocytes communicate with B-cells via BAFF and BAFFR, so that B-cells are stimulated, but BAFF is also produced to stimulate cells in autocrine way. The decrease of BAFFR expression in SjS patients suggests that there is the mechanism that attempts to take over in order to balance the high level of BAFF.
\end{abstract}

Keywords: Sjögren's syndrome; BAFF; BAFFR; intracellular expression; $s B A F F$

\section{Introduction}

Sjögren's syndrome $(\mathrm{SjS})$ is recognized as autoimmune exocrinopathy that primarily affects middle aged females $(10,12)$. Pathogenesis of $\mathrm{SjS}$ is caused by the immune system abnormalities. The exocrine glands are inflamed in $\mathrm{SjS}$ patients. Focal lymphocytic infiltrates surrounding the tubular epithelium are the hallmark of this inflammation. B-cell activity is excessive, resulting in wide spectrum of autoantibodies production (19). The pathogenesis of $\mathrm{SjS}$ still remains elusive with previous emphasis on the pathogenetic role of T cells. Current data indicates the substantial contribution of B-cells in the immunopathogenesis of $\mathrm{SjS}$ (1). B-cells may constitute germinal centers in affected salivary glands. B-cells can act as antigen presenting cells, in this way fuelling abnormal $\mathrm{T}$ cells response. B-cells produce not only antibodies, but also several cytokines. Numerous cytokines are indispensable for B-cell functions. Among them, the most important role is deserved to B-cell activating factor belonging to the TNF $\alpha$ family (BAFF). The cytokine BAFF plays a key role in B-cell differentiation, survival, and activation (16). BAFF is a ligand for three membrane receptors BCMA (B-cell maturation receptor), TACI (transmembrane activator and calcium modulator and cyclophilin ligand interactor), and BAFFR the latter receptor is being chiefly involved in BAFF sig- naling (11). Cells of innate immune system, including monocytes, macrophages, neutrophils, and dendritic cells, are the main producers of BAFF. Most recently, other cells that produce BAFF have been identified. These cells are of nonhematopoietic origin and include some types of epithelial cells, osteoclast, and astrocytes in CNS (6). BAFFR is widely expressed on B-cells. As for non-B-cells, BAFFR is upregulated on activated T cells and constitutively expressed on Treg subset of T cells (21). It was predicted that excessive production of BAFF shall break B-cell self-tolerance and allow self-reactive B-cells to survive. BAFF transgenic mice produce autoantibodies, leading to salivary gland destruction, the feature reminiscent of SjS (9). The serum level of soluble sBAFF is elevated in SjS patients. In addition, there is increased expression of BAFF on blood mononuclear cells (1).

Numerous biological therapies have been already approved or are in the development. These therapies aim the abnormally active soluble or membrane molecules, whose presence is a characteristic of autoimmune diseases, such as systemic lupus, rheumatoid arthritis, as well as secondary Sjögren's syndrome. Several B-cell molecules can be targeted. The most widely studied target for achieving B-cell depletion is CD20 molecule found on pre-B and mature B-cells. Two different types of BAFF antagonist are at various stages of clinical development (3). Our study is in accord 
with overall effort that shows further roles of BAFF/BAFFR in the immunopathogenesis of $\mathrm{SjS}$. We evaluated the expression of BAFF/BAFFR in peripheral blood monocytes and B-cells of SjS patients using flow cytometry and we also measured sBAFF in serum.

\section{Patients and Controls}

19 patients with SjS participated in this study. All of them were diagnosed with primary $\mathrm{SjS}$. All patients fulfilled the European-American consensus group criteria (AECC). Diagnosis of Sjögren's syndrome was based on routine evaluation of patient's symptoms and laboratory results (autoantibodies analysis, test of salivary flow rate, Schirmer's test, medical records, etc.) performed at the Departments of Dentistry, Immunology and Allergy, Rheumatology and Ophthalmology at the University Hospital in Hradec Králové, Czech Republic. The control group enrolled in this study used no medication and consisted of 21 sex and age-matched individuals from the same geographical area. All participants confirmed their participation in the study by a written consent. The study project was approved by the Ethics Committee of the University Hospital in Hradec Králové, Czech Republic.

Tab. 1: Demographic and clinical data.

\begin{tabular}{|l|c|c|}
\hline & Controls (20) & Patients (19) \\
\hline Men/Women (n) & $1 / 19$ & $1 / 18$ \\
\hline Age (years) & 54 & 55 \\
\hline Symptoms of xerophthalmia (n) & 0 & 17 \\
\hline Symptoms of xerostomia (n) & 0 & 19 \\
\hline Dysphagia & 0 & 14 \\
\hline Joint pain (n) & 0 & 16 \\
\hline Thyropathy (n) & 0 & 3 \\
\hline Autoantibodies (n) & 0 & 15 \\
\hline NSAIDs (n) & 0 & 4 \\
\hline Cystosporin A (n) & 0 & 3 \\
\hline Corticosteroids (n) & 0 & 4 \\
\hline Antimalarics (n) & 0 & 1 \\
\hline
\end{tabular}

\section{Materials and Methods}

Peripheral venous blood was collected into Vacutainer lithium heparin tubes (BD, UK). Mononuclear cells were separated from Hank's-diluted blood sample (1:1) layered over Histopaque-1077 (Sigma-Aldrich, CR). Separated cells were washed twice with PBS containing 2\% FBS and 1 mM EDTA (Sigma-Aldrich, CR). Cell number was counted with hemocytometer and monoclonal antibodies were added accordingly. Monoclonal antibodies against human
BAFF FITC (clone 1D6) and BAFFR PE (clone 8A7) were purchased from eBioscience, (UK) while anti-human CD14 PerCP (clone MEM-15) and anti-CD19 APC (clone LT19) antibodies were bought from Exbio, CR. Following incubation, cells were fixed with $1 \%$ paraformaldehyde for $15 \mathrm{~min}$ and washed. Prior to intracellular staining with antibodies (anti-BAFF, anti-BAFFR), cells were permeabilized with $0.5 \%$ saponin $(5 \mathrm{~min})$. Cells were washed again and measured immediately with CellQuest software on FACSCalibur (BD, USA). At least a minimum of 30,000 cells was acquired. Instrument setting and compensation was regularly adjusted using Calibrite beads with FACSComp software (BD, USA).

FlowJo software (TreeStar, USA) was used for analysis of data acquired by flow cytometry. Monocytes and B cells were distinguished on the basis of a presence of CD14 and CD19, respectively (Fig. 1). Expression of BAFF and BAFF receptor was characterized by median fluorescence intensity (MFI) that was further used for statistical analysis.

Histogram charts contain examples of flow cytometry data to show the differences between control group and group of patients. Every example that we selected was of value close to the median that described a measured parameter in a given group.

Vacutainer tubes with a thrombin additive (BD, UK) were used to collect serum samples. These samples were stored at $-70{ }^{\circ} \mathrm{C}$. sBAFF was detected using anti-human sBAFF ELISA kit purchased from R\&D Systems (USA). Sensitivity of the assay was $2.86 \mathrm{pg} / \mathrm{mL}$. Concentration of sBAFF was measured on MRX microplate reader using Revelation software (Dynatech Laboratories, USA).

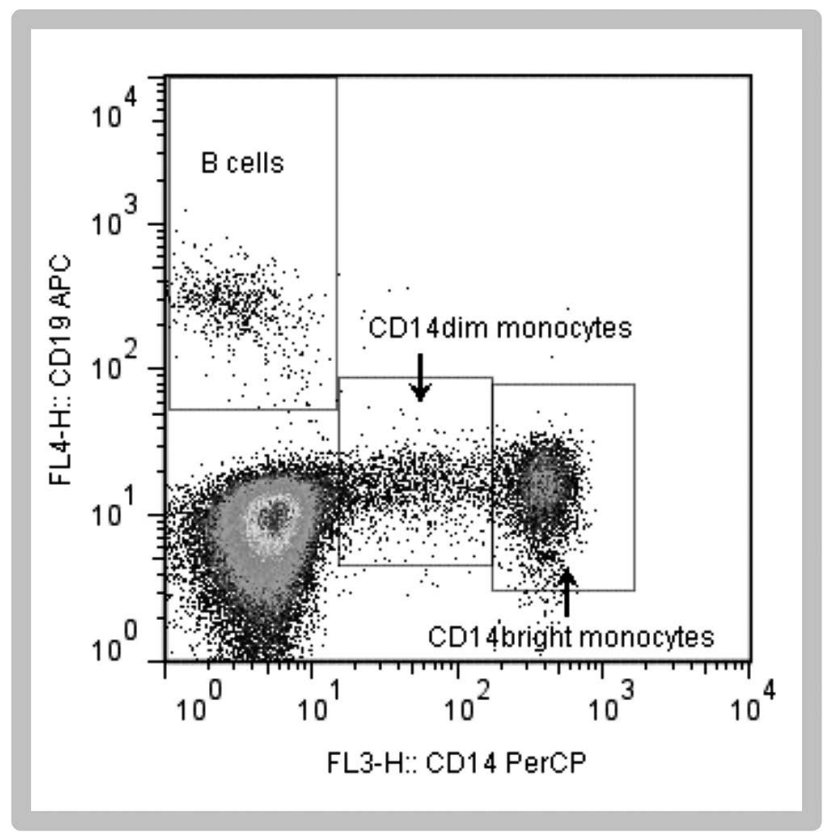

Fig. 1: Populations of cells in which expression of BAFF and BAFFR was further analyzed. 


\section{Statistical analysis}

Expression of BAFF and BAFFR, and percentage of cell populations was compared between patients and controls. For this purpose, t-test or non-parametric tests (Man-Whitney test and Kolmogorov-Smirnov test) were used. Differences between clinical and demographic data were tested using $\chi^{2}$ test and Fisher exact test. Before all these comparisons, normality of data sets was tested with Shapiro-Wilks test. Homoscedasticity was determined using Levene's test. All tests were performed at the 5\% significance level.
Plots display median values, quartiles (box), and ranges of non-outlier values (whiskers). If not stated otherwise, any value in the manuscript represents median.

\section{Results}

Similar number of mononuclear cells was isolated from patients and controls (data not shown). When compared to control group, there was the prevalence of lymphocytes in isolated mononuclear cells of SjS patients. This difference was statistically significant (83\% in control group, $87 \%$ in SjS patients, $\mathrm{p}<0.05)$. However, the percentage

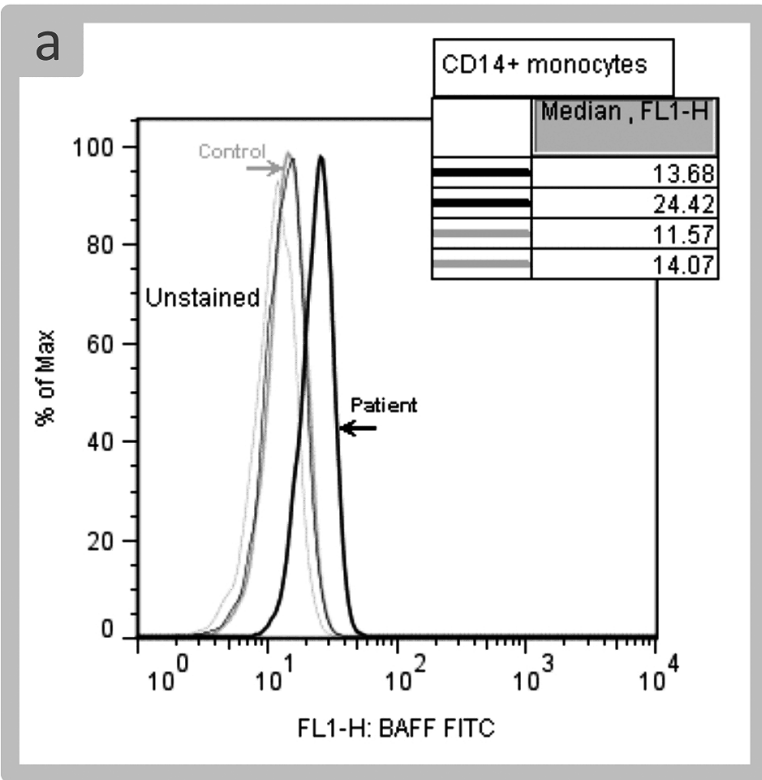

\section{b}
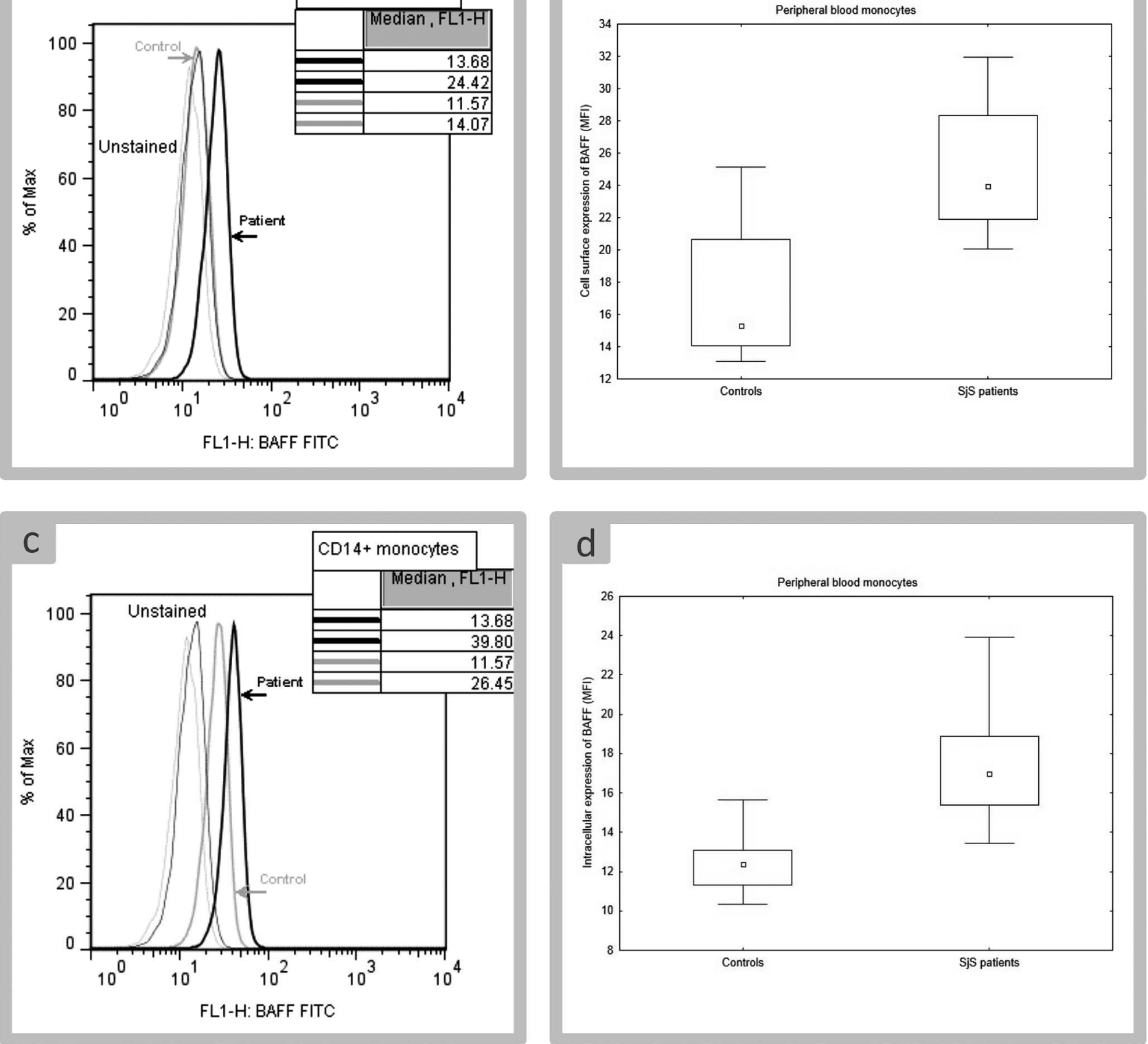

Fig. 2: Comparison between controls' and patients' BAFF expression on monocytes. 
of B cells did not significantly differ between both groups ( $1.27 \%$ in controls and $1.71 \%$ in patients). The percentage of monocytes was higher in control group (16.2\%) than in SjS patients $(11.5 \%, \mathrm{p}<0.05)$. When monocytes were considered as $100 \%$ population, the percentage of monocyte subpopulations (CD14dim, CD14brigh) was not different between controls and patients.

Cell surface expression of BAFF was very low on healthy monocytes, making the increased expression in SjS patient distinctive from control group (Fig. 2A). The difference between both groups was statistically significant (Fig. 2B). BAFF was also localized intracellularly (Fig. 2C). Similarly to cell surface BAFF, intracellular
BAFF was significantly increased in monocytes of $\mathrm{SjS}$ patients (Fig. 2C).

There was no difference in expression of BAFF between CD14dim and CD14bright monocytes, therefore, monocytes were analyzed as a unified population.

As in case of monocytes, cell surface and intracellular expression of BAFF was significantly increased in patients' B cells (Fig. 3). However, no correlation between increased BAFF on monocytes and increased BAFF on B cells was found. There was also no correlation in intracellular BAFF (data not shown).

Serum concentration of sBAFF displayed a wide range of values in patients and significantly differed from the concen-
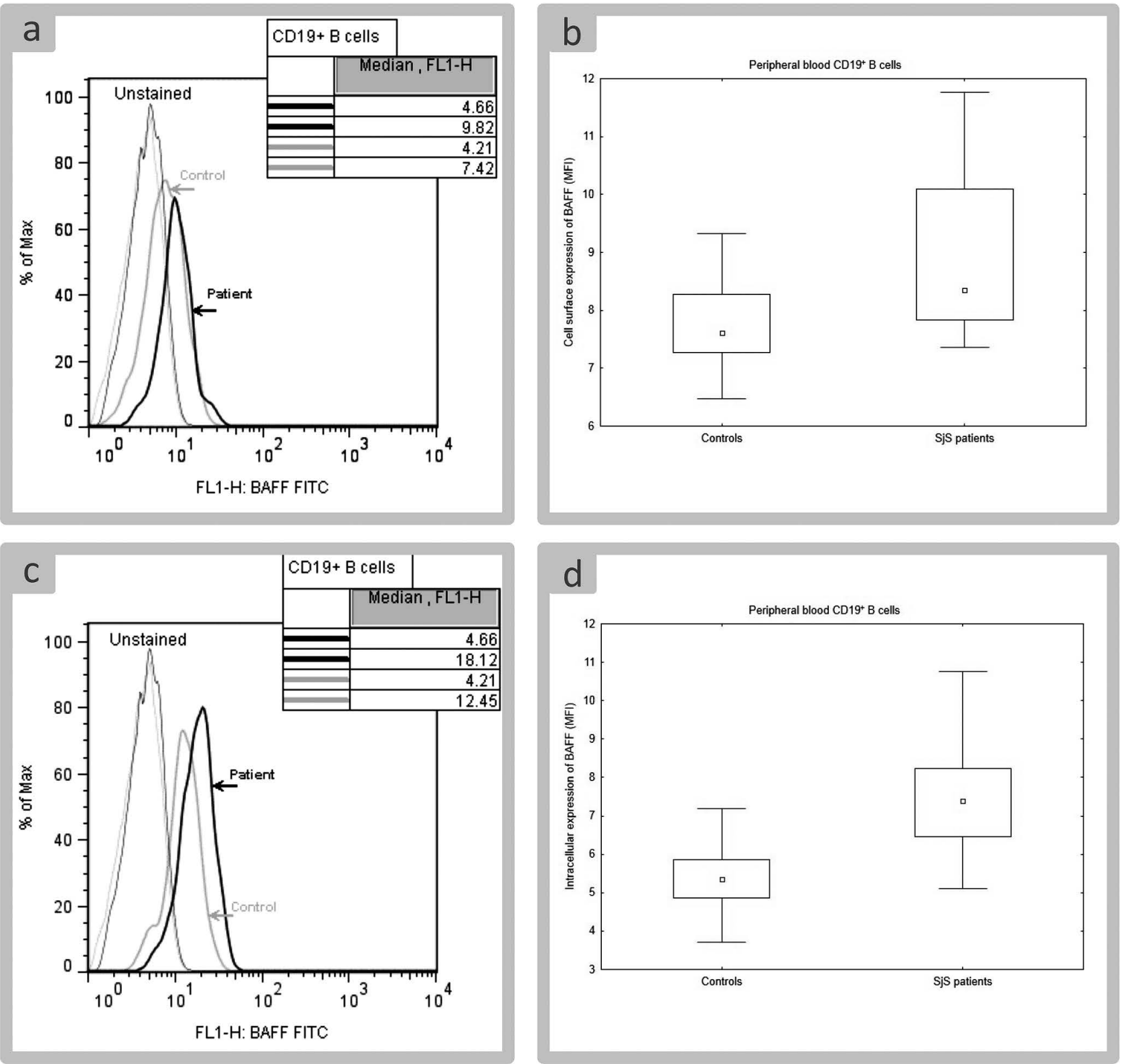

Fig. 3: Comparison between controls' and patients' BAFF expression on B cells. 


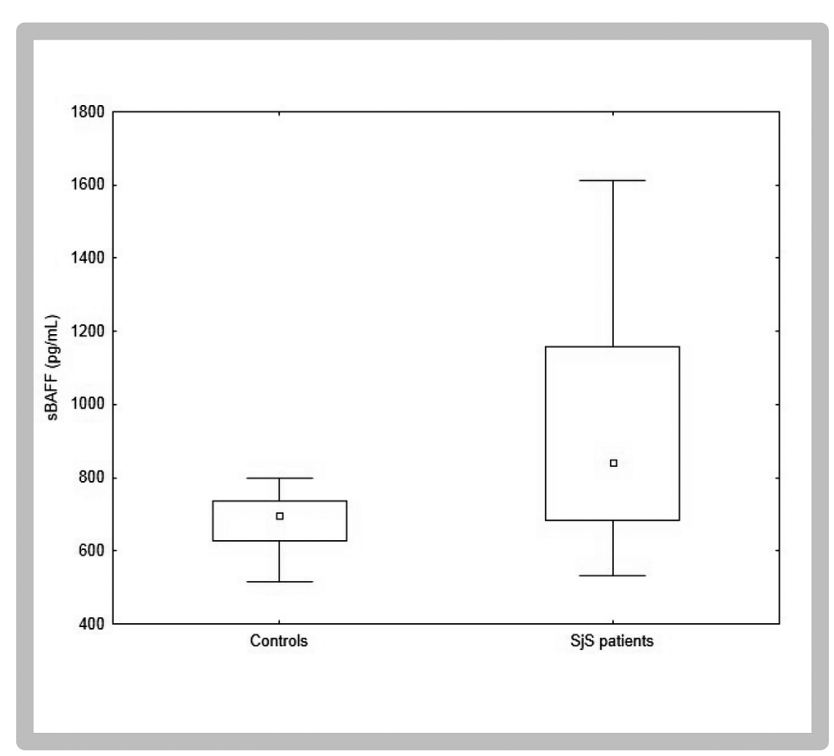

Fig. 4: Comparison of sBAFF concentration between controls and SjS patients.

tration in control group (Fig. 4). Patients who expressed high BAFF either on monocytes or B cells did not necessarily have high concentration of sBAFF; no correlation was observed between SBAFF and cell-bound BAFF (data not shown).

Expression of cell surface BAFFR on B cells was surprisingly decreased in SjS patients (Fig. 5A, B). High expression of BAFFR was also found intracellularly, but there was no difference in intracellular BAFFR between controls and patients (data not shown).

Cell surface BAFFR was expressed on monocytes, however, intracellular localization of BAFFR was not observed in this population of cells. Distribution of BAFFR values differed significantly between control group and patients (Fig. 5C). Nonetheless, due to the fact that patients' data set completely overlapped with controls, it was not possible to conclude that expression of BAFFR was increased on monocytes of $\mathrm{SjS}$ patients.

\section{Discussion}

The main histopathological feature of $\mathrm{SjS}$ is the periductal cellular infiltration of the salivary glands, predominantly by T-cells, whilst B-cells and plasma cells are commonly found in more severe lesions. Although innate immunity cells, such as monocytes, macrophages, and dendritic cells, constitute less than $5 \%$ of the total infiltrating population, they play an important role in the pathogenesis of SjS (20). Mounting evidence supports the idea that BAFF/ BAFFR pathways are critically involved in abnormal B-cells activity typically seen in SjS patients. This abnormal activity demonstrates as hypergammaglobulinemia, presence of a broad spectrum of autoantibodies, ectopic germinal centre formation, oligoclonal B-cell expansion, and the well
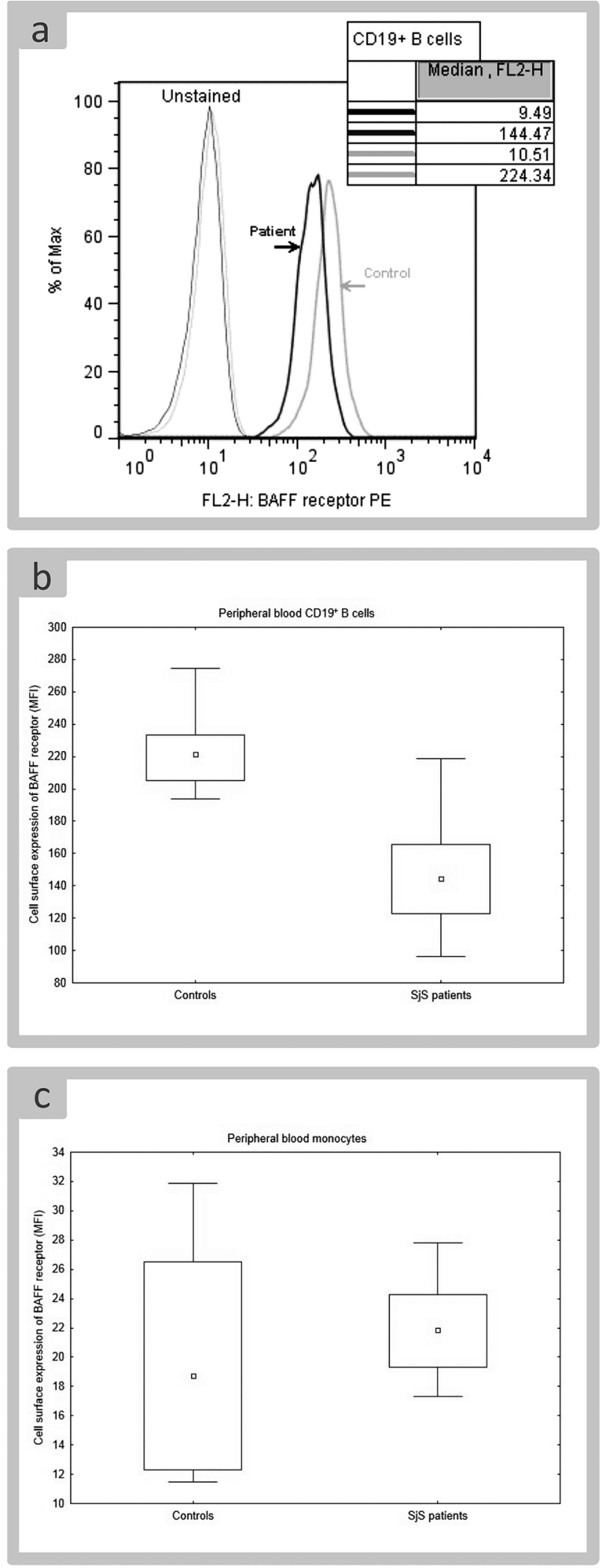

Fig. 5: Differences in cell surface expression of BAFFR. 
documented risk of B-NHL lymphoma development. There are consistent reports that monocytes are contributing to the elevated of BAFF in SjS patients (8). Serum level of BAFF in $\mathrm{SjS}$ patients correlates with BAFF mRNA expression (2). Peripheral blood monocytes of SjS patients produce significantly higher amount of SBAFF in vitro both spontaneously and after stimulation with interferon $\gamma(22)$. We found significantly higher presence of BAFF both on the surface and in the intracellular compartment of SjS patients' monocytes when compared to healthy controls. BAFF can contribute to B-cells physiology via its binding to the three membrane receptors of TNF $\alpha$ receptor family: BCMA, TACI, and BR3. BAFF interacts chiefly with BR3 which is for this reason designated as BAFFR. BAFFR is expressed on B-cells, activated T-cells, and regulatory T-cells. The expression of BAFFR is increasing as B-cells mature (21). We detected low-level expression of this receptor on monocytes of $\mathrm{SjS}$ patients, but no significant differences between patients and healthy control were found. In addition, no intracellular BAFFR in monocytes was identified in our study.

It is now firmly established that abnormal production of BAFF in SjS patients is linked to enhanced activity of type I interferon system (11). Increased expression of interferons I - regulated genes was described in the salivary glands, in which plasmocytoid dendritic cells were found as a principal source of interferon $\alpha$. This can represent the link between innate and adaptive immunity in $\mathrm{SjS}(6)$. The interferons type I signature in CD14 monocytes along with higher BAFF mRNA expression identifies SjS patients with higher clinical activity of the disease (2). BAFF is a TNF $\alpha$-like cytokine essential for the maturation and survival of peripheral B-cells. It exists in a membrane bound and secreted form. Cell sources of BAFF include dendritic cells, macrophages, neutrophils, activated T-cells and B-cells (4). We detected significantly enhanced membrane expression of BAFF on CD19 B-cells in blood of patients with SjS compared to healthy controls. The elevated presence of BAFF was also found in cytoplasma of $\mathrm{SjS}$ patients B-cells. BAFFR expression on B-cells of our patients was decreased. This is in accord with other study with no difference between naive and memory B-cells. The BAFFR down-regulation correlated with BAFF level and could be reproduced ex vivo by long-term exposure of B-cell to BAFF. The decrease of BAFFR expression on B-cells was greater in patients with extraglandular involvement than in patients with glandular involvement only (15). The decrease in the expression of BAFFR on B-cells could be explained by very likely internalization of BAFF-occupied BAFFR.

Serum level of sBAFF displayed a wide range of values in our patients and was significantly higher compared to controls. Patients who showed high expression of BAFF either on B-cells or monocytes did not necessarily have high concentration of sBAFF.

We observed no correlation between sBAFF and cell bound BAFF. Conflicting results exists for BAFF quantification. Furthermore, concentration of SBAFF fluctuates due to changes in inflammatory activity and extent of the disease, as well as the type of treatment $(13,17)$. In-house assays are used by some research groups. We used commercially available kit to detect sBAFF and our results are comparable with results of Quartuccio et al. (14) who reported upregulation of sBAFF in $\mathrm{SjS}$ patients and significant association of elevated sBAFF with development of B-cell lymphoproliferative disease.

In conclusion, BAFF controls B-cell proliferation survival, and maturation (18). BAFF is mainly produced by monocytes and excerts its function trough BAFFR. BAFFR signaling involves canonical and non-canonical NFKB pathways (5) but there is evidence that BAFFR induces other pathways (7). Our results indicate that activated monocytes communicate with B-cells via BAFF and BAFFR, so that B-cells are stimulated, but BAFF is also produced to stimulate cells in autocrine way. The decrease of BAFFR expression in $\mathrm{SjS}$ patients suggest that there is a mechanisms that attempts to take over in order to balance the high level of BAFF. Since the level of BAFF is still high in $\mathrm{SjS}$ patient's B-cells, therapy targeting BAFF is likely to bring benefits to these patients.

\section{Dedication}

This paper is dedicated to celebrate 70th anniversary of foundation of Faculty of Medicine in Hradec Králové, Charles University in Prague, Czech Republic.

\section{Acknowledgements}

This work was supported by Charles University in Prague, Faculty of Medicine in Hradec Králové, Czech Republic, project "PRVOUK" P37/10.

The authors declare no conflict of interest.

\section{References}

1. Bowman S, Barone F. Biologic treatments in Sjögren's syndrome. Presse Med 2012; 41: e495-e509.

2. Brkic Z, Maria NI, van Helden-Meeuwsen CG et al. Prevalence of interferon type I signature in CD14 monocytes of patients with Sjögren's syndrome and association with disease activity and BAFF gene expression. Ann Rheum Dis 2013; 72(5): $728-735$.

3. Cornec D, Devauchelle-Pensec V, Tobón GJ, Pers JO, Jousse-Joulin S, Saraux A. B cells in Sjögren's syndrome: from pathophysiology to diagnosis and treatment. J Autoimmun 2012; 39(3): 161-167.

4. Daridon C, Devauchelle V, Hutin P et al. Aberrant expression of BAFF by $\mathrm{B}$ lymphocytes infiltrating the salivary glands of patients with primary Sjögren's syndrome. Arthritis Rheum 2007; 56(4): 1134-1144.

5. Gardam S, Brink R. Non-canonical NF- $\mathrm{kB}$ signaling initiated by BAFF influences B cell biology at multiple junctures. Front Immunol 2014; 4(509): eCollection 2014.

6. Gottenberg JE, Cagnard N, Lucchesi C et al. Activation of IFN pathways and plasmacytoid dendritic cell recruitment in target organs of primary Sjögren's syndrome. Proc Natl Acad Sci USA 2006; 103(8): 2770-2775.

7. Groom J, Mackay F. B cells flying solo. Immunol Cell Biol 2008; 86(1): 40-46.

8. Lavie F, Miceli-Richard C, Ittah M, Sellam J, Gottenberg JE, Mariette X. B-cell activating factor of the tumour necrosis factor family expression in blood monocytes and T cells from patients with primary Sjögren's syndrome. Scand J Immunol 2008; 67(2): 185-192. 
9. Mackay F, Woodcock SA, Lawton P et al. Mice transgenic for BAFF develop lymphocytic disorders along with autoimmune manifestations. J Exp Med 1999; 190 (11): 1697-1710.

10. Moutsopoulos HM. Sjögren's syndrome: autoimmune epithelitis. Clin Immunol Immunopathol 1994; 72(2): 162-165.

11. Nikolov NP, Illei GG. Pathogenesis of Sjögren's syndrome. Curr Opin Rheumatol 2009; 21(5): 465-470.

12. Pers JO, Youinou P. Are the B cells cast with the leading part in the Sjögren's syndrome scenario? Oral Dis 2014; 20(6): 529-537; doi: 10.1111/odi.12153.

13. Pollard RPE, Abdulahad WH, Vissink A, et al. Serum levels of BAFF, but not APRIL, are increased after rituximab treatment in patients with primary Sjögren's syndrome: data from a placebo-controlled clinical trial. Ann Rheum Dis 2013; 72(1): 146-148

14. Quartuccio L, Salvin S, Fabris M et al. BLyS upregulation in Sjögren's syndrome associated with lymphoproliferative disorders, higher ESSDAI score and B-cell clona expansion in the salivary glands. Rheumatology (Oxford) 2013; 52(2): 276-281.

15. Sellam J, Miceli-Richard C, Gottenberg JE et al. Decreased B cell activating facto receptor expression on peripheral lymphocytes associated with increased disease activity in primary Sjögren's syndrome and systemic lupus erythematosus. Ann Rheum Dis 2007; 66(6): 790-797.
16. Schenider P, MacKay F, Steiner V et al. BAFF, a novel ligand of the tumor necrosis factor family, stimulates B cell growth. J Exp Med 1999; 189(11): $1747-1756$

17. Varin MM, Le Pottier L, Youinou P, Saulep D, Mackay F, Pers JO. B-cell tolerance breakdown in Sjögren's syndrome: focus on BAFF. Autoimmun Rev 2010; 9(9): 604-608.

18. Vincent FB, Sulep-Easton D, Figgett WA, Fairfax KA, Mackay F. The BAFF/ APRIL system: emerging functions beyond B cell biology and autoimmunity. Cytokine Growth Factor Rev 2013; 24(3): 203-215.

19. Vitali C, Bombardieri S, Jonsson R et al. Classification criteria for Sjögren's syndrome. A revised version of the European criteria proposed by the American-European Consensus Group. Ann Rheum Dis 2002; 61(6): 554-558.

20. Voulgarelis M, Tzioufas AG. Current aspects of pathogenesis in Sjögren's syndrome. Ther Adv Musculoskelet Dis 2010; 2(6): 325-334

21. Ye Q, Wang L, Wells AD et al. BAFF binding to T cell-expressed BAFF-R costimulates T cell proliferation and alloresponses. Eur J Immunol 2004; 34(10): $2750-2759$.

22. Yoshimoto K, Tanaka M, Kojima M et al. Regulatory mechanisms for the production of BAFF and IL- 6 are impaired in monocytes of patients of primary Sjögren's syndrome. Arthritis Res Ther 2011; 13(5): R170.

Received: 22/01/2015

Accepted in revised form: 01/04/2015

\section{Corresponding author:}

Jan Krejsek, Department of Clinical Immunology and Allergology, University Hospital in Hradec Králové, 500 05, Hradec Králové, Czech Republic; e-mail: jan.krejsek@fnhk.cz 